\title{
Synthesis of tert-Butyl 1,3-Diaryl-3-oxopropylcarbamates by a Regiocontrolled Reduction of Ketoaziridines
}

\author{
Heshmat A. Samimi*a \\ Bohari M. Yamin \\ Fatemeh Saberia \\ a Faculty of Science, Department of Chemistry, Shahrekord \\ University, PO Box 115, Shahrekord, Iran \\ samimi-h@sci.sku.ac.ir \\ b School of Chemical Sciences and Food Technology, University \\ Kebangsaan Malaysia, UKM 43500 Bangi Selangor, Malaysia
}
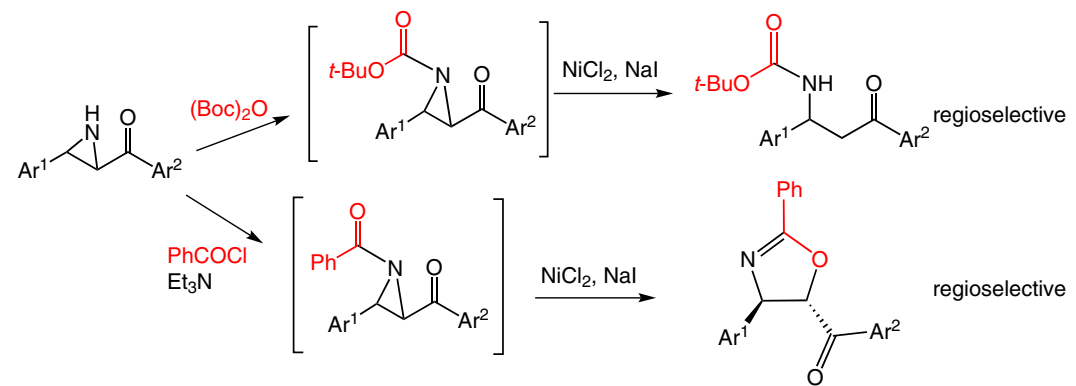

Received: 03.07.2014

Accepted after revision: 06.08.2014

Published online: 17.09 .2014

DOI: 10.1055/s-0034-1379029; Art ID: ss-2014-z0402-op

Abstract A new, convenient approach for the reductive ring opening of $\mathrm{N}$-H ketoaziridines is described. Treatment of $\mathrm{N}-\mathrm{H}$ ketoaziridines with di-tert-butyl dicarbonate $\left[(\mathrm{Boc})_{2} \mathrm{O}\right]$ in the presence of sodium iodide and nickel(II) chloride results in the corresponding tert-butyl 1,3-diaryl3-oxopropylcarbamates by a regiocontrolled reaction. The structure of the regioisomeric product was confirmed by $\mathrm{X}$-ray crystal structure analysis.

Key words aziridines, regioselectivity, ring opening, reduction, nickel(II) chloride

The reductive ring opening of aziridines is a synthetically useful transformation for the preparation of amino compounds, especially in the synthesis of $\beta$-amino ketones, ${ }^{1,2}$ which are important for the synthesis of biologically active compounds. ${ }^{3-7}$

Several methods are known for the synthesis of $\beta$-amino ketones from the ring opening of aziridines. A literature survey shows the few reducing agents, including Raney nickel in ethanol, ${ }^{8}$ Pearlman's catalyst $\left[\mathrm{Pd}(\mathrm{OH})_{2} / \mathrm{C}\right],{ }^{9}$ Adam's catalyst $\left(\mathrm{PtO}_{2} / \mathrm{HCO}_{2} \mathrm{H}\right),{ }^{10}$ sodium borohydride, ${ }^{11}$ tributyltin hydride, ${ }^{12}$ poly(methylhydrosiloxane) (PMHS), ${ }^{13}$ silyllithium reagents, ${ }^{14}$ magnesium ${ }^{15}$ and lithium ${ }^{16}$ metal reagents, titanium tetraiodide, ${ }^{17}$ samarium(II) iodide $^{18}$ and visible-light photoredox ruthenium catalysts, ${ }^{19}$ for the reductive ring opening of aziridines. None of these methods, however, result in a direct reduction reaction of $\mathrm{N}-\mathrm{H}$ aziridines to give derivatives of $\beta$-carbamato ketones.

Our recent interest in the ring opening and ring expansion of ketoaziridines motivated us to synthesize tert-butyl 1,3-diaryl-3-oxopropylcarbamates. ${ }^{20-27}$
Previous reports have shown that replacement of the hydrogen of the $\mathrm{N}-\mathrm{H}$ moiety with an electron-withdrawing substituent increases the susceptibility of $\mathrm{N}-\mathrm{H}$ aziridines to ring-opening or ring-enlargement reactions. ${ }^{28-30}$ So, our first aim was to synthesize $N$-Boc-substituted ketoaziridines as a precursor for preparation of the corresponding nitrogen-containing compounds via ring opening or ring enlargement. At first, we investigated the reaction of ditert-butyl dicarbonate $\left[(\mathrm{Boc})_{2} \mathrm{O}\right]$ and 2-(4-chlorobenzoyl)3-(4-chlorophenyl)aziridine (1a) in chloroform, acetonitrile, ethanol or acetone in the presence of triethylamine. The mixture was stirred for 10 hours under refluxing conditions, but 1a was recovered unchanged (Table 1, entries 14); none of the desired product was detected.

Based on the well-documented transformation of $\mathrm{N}$ acyl- or $N$-Boc-substituted aziridines into oxazolines in the literature ${ }^{31-34}$ and our success ${ }^{20,21}$ with the ring-expansion reaction of $\mathrm{N}$-acyl-substituted aziridines with sodium iodide, we envisioned that ring expansion of 1a with (Boc) $)_{2} \mathrm{O}$ in the presence of sodium iodide might be similarly achieved to give oxazolidin-2-one $\mathbf{6} \mathbf{a}$ in a one-pot reaction. Thus, we examined the reaction of aziridine $\mathbf{1 a}$ with $(\mathrm{Boc})_{2} \mathrm{O}$ in the presence of sodium iodide in acetone for achieving this aim; however, no reaction occurred, even under refluxing conditions (Table 1 , entry 5 ).

In order to evaluate the effect of a Lewis acid in this reaction, we tried the reaction of 2-(4-chlorobenzoyl)-3-(4chlorophenyl)aziridine (1a) with (Boc) $)_{2} \mathrm{O}$ in the presence of sodium iodide and some Lewis acids $\left(\mathrm{ZnCl}_{2}, \mathrm{CuCl}_{2}\right)$ in refluxing acetone; with zinc chloride and copper(II) chloride, a new product $\mathbf{4 a}$ was obtained in low yields (Table 1, entries 6 and 7).

In another attempt, we examined the reaction of 1a with (Boc) $)_{2} \mathrm{O}$ in the presence of nickel(II) chloride and sodium iodide (1 mmol) (Table 1 , entry 8$)$. At this stage, thinlayer chromatography confirmed the formation of a new 
Table 1 Optimization of the Conditions for the Reaction of Aziridine $1 \mathrm{a}$ with $(\mathrm{Boc})_{2} \mathrm{O}^{\mathrm{a}}$

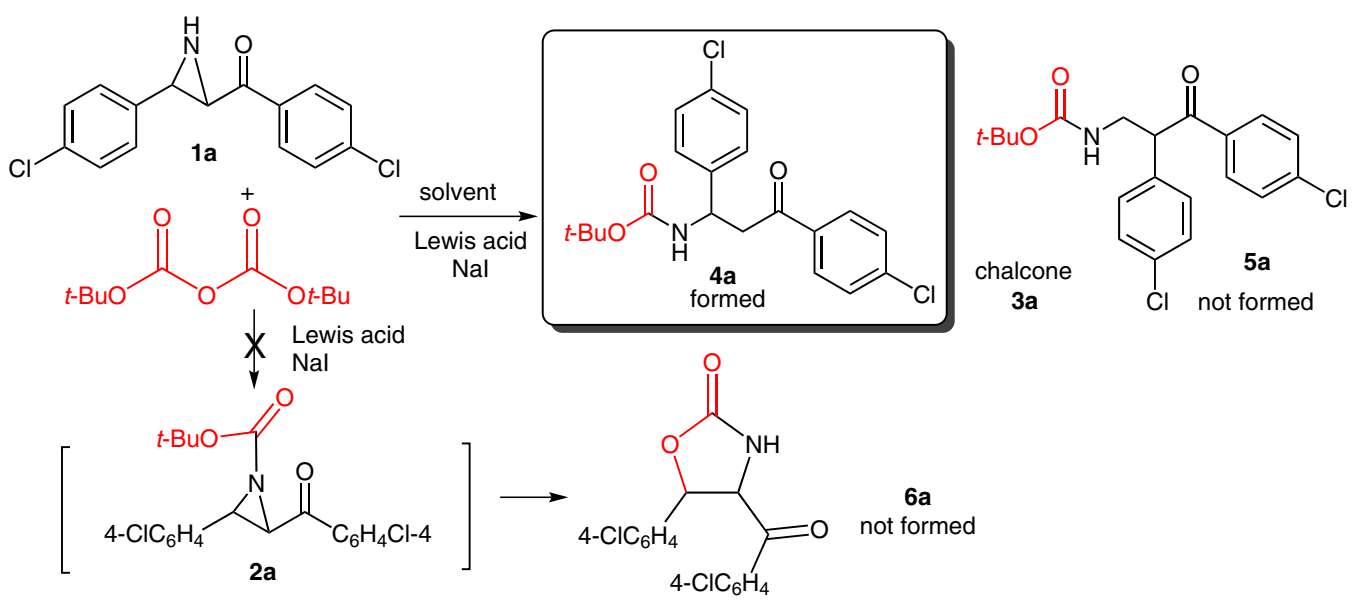

\begin{tabular}{|c|c|c|c|c|c|}
\hline \multirow[t]{2}{*}{ Entry } & \multirow[t]{2}{*}{ Reagent } & \multirow[t]{2}{*}{ Solvent } & \multirow[t]{2}{*}{ Time (h) } & \multicolumn{2}{|c|}{ Yield ${ }^{\mathrm{b}}(\%)$} \\
\hline & & & & $3 a$ & $4 a$ \\
\hline $1^{c}$ & $\mathrm{Et}_{3} \mathrm{~N}$ & $\mathrm{CHCl}_{3}$ & 10 & 0 & 0 \\
\hline $2^{c}$ & $\mathrm{Et}_{3} \mathrm{~N}$ & $\mathrm{MeCN}$ & 10 & 0 & 0 \\
\hline $3^{c}$ & $\mathrm{Et}_{3} \mathrm{~N}$ & $\mathrm{EtOH}$ & 10 & 0 & 0 \\
\hline $4^{c}$ & $\mathrm{Et}_{3} \mathrm{~N}$ & acetone & 10 & 0 & 0 \\
\hline $5^{c}$ & $\mathrm{Nal}$ & acetone & 6 & 0 & 0 \\
\hline 6 & $\mathrm{Nal}, \mathrm{ZnCl}_{2}$ & acetone & 8 & 0 & 16 \\
\hline 7 & $\mathrm{Nal}, \mathrm{CuCl}_{2}$ & acetone & 8 & 0 & 23 \\
\hline 8 & $\mathrm{Nal}, \mathrm{NiCl}_{2}$ & acetone & 8 & 19 & 71 \\
\hline $9^{c}$ & $\mathrm{ZnCl}_{2}$ & acetone & 10 & 0 & 0 \\
\hline $10^{c}$ & $\mathrm{CuCl}_{2}$ & acetone & 10 & 0 & 0 \\
\hline 11 & $\mathrm{NiCl}_{2}$ & acetone & 10 & 44 & 0 \\
\hline
\end{tabular}

a $\mathbf{1 a} /(\mathrm{Boc})_{2} \mathrm{O} /$ reagent $=1: 1: 1$.

$\mathrm{b}$ Isolated yields.

c The starting material was recovered.

compound, along with the corresponding chalcone $\mathbf{3 a}$. The crude product was purified by column chromatography, surprisingly to provide tert-butyl 1,3-bis(4-chlorophenyl)3-oxopropylcarbamate (4a) or tert-butyl 2,3-bis(4-chlorophenyl)-3-oxopropylcarbamate (5a). Since the spectroscopic data were not conclusive for $\mathbf{4 a}$ or $\mathbf{5 a}$, X-ray crystallographic analysis was conducted to verify the product structure as $\mathbf{4 a}$ (Figure 1).

It is striking to note that X-ray crystal structure analysis of the representative product $\mathbf{4 a}$ (Figure 1) confirms the regiocontrolling nature of this reaction. The reaction proceeded with selective ring opening at the $\mathrm{C}-\mathrm{N}$ bond $\alpha$ to the benzoyl moiety, whereas the $\mathrm{C}-\mathrm{N}$ bond of the benzyl group was not cleaved. The same stereochemistry has been generalized for all other products formed from this reaction.
A further examination of the reaction of $\mathbf{1 a}$ and (Boc) $)_{2} \mathrm{O}$ with zinc chloride, copper(II) chloride or nickel(II) chloride in the absence of sodium iodide failed to give any product 4a (Table 1, entries 9-11).

To expand the scope of this novel method, several substituted ketoaziridines 1 were reacted with (Boc) $)_{2} \mathrm{O}$ in the presence of nickel(II) chloride and sodium iodide, resulting in production of the tert-butyl 1,3-diaryl-3-oxopropylcarbamates 4 in moderate to good yields (Table 2). All the products were characterized by ${ }^{1} \mathrm{H}$ NMR, ${ }^{13} \mathrm{C}$ NMR and IR spectroscopy.

We have previously reported the mechanism of the iodide ion catalyzed isomerization of $\mathrm{N}$-acyl-substituted aziridines by attack of the nucleophile at C-2 of the aziridine and subsequent cyclization to the corresponding oxazolines. ${ }^{20}$ We have now found that the action of nickel(II) chloride with sodium iodide on 2-aroyl-3-arylaziridines is 


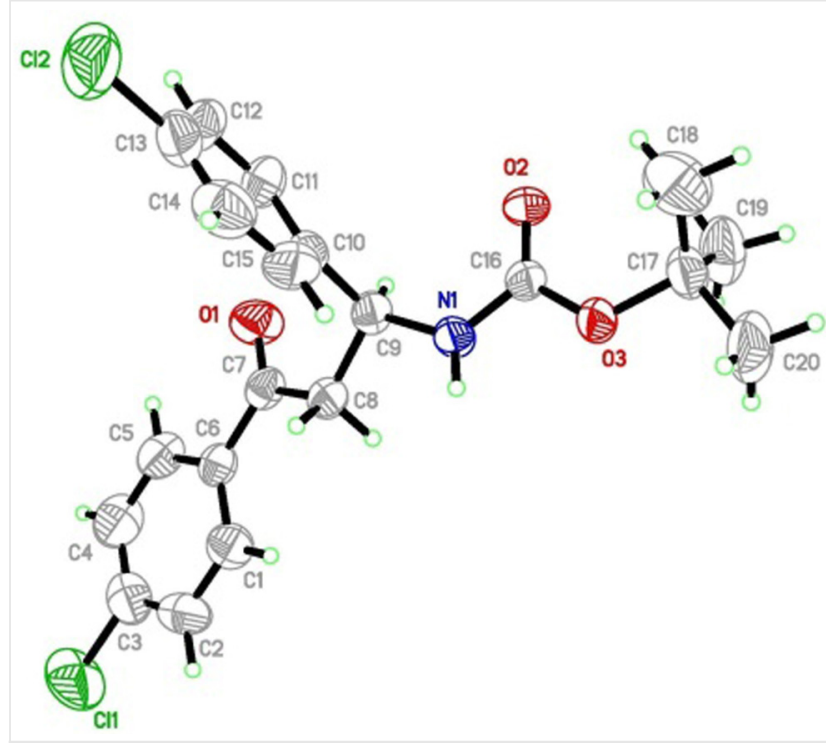

Figure 1 ORTEP view of the crystal structure of $4 a$

Table 2 Substrate Scope for the Reductive Ring Opening of N-H Ketoaziridines 1 to the Corresponding tert-Butyl 1,3-Diaryl-3-oxopropylcarbamates $\mathbf{4}^{\text {a }}$

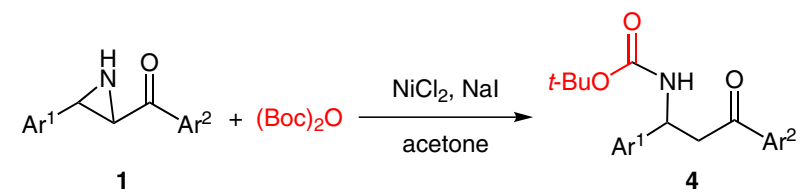

\begin{tabular}{lllll}
\hline Product & $\mathrm{Ar}^{1}$ & $\mathrm{Ar}^{2}$ & Time (h) & Yield $^{\mathrm{b}}(\%)$ \\
\hline 4a & $4-\mathrm{ClC}_{6} \mathrm{H}_{4}$ & $4-\mathrm{ClC}_{6} \mathrm{H}_{4}$ & 8 & 71 \\
4b & $3-\mathrm{O}_{2} \mathrm{NC}_{6} \mathrm{H}_{3}$ & $\mathrm{Ph}$ & 10 & 68 \\
4c & $4-\mathrm{ClC}_{6} \mathrm{H}_{4}$ & $\mathrm{Ph}$ & 8 & 73 \\
4d & $\mathrm{Ph}$ & $\mathrm{Ph}$ & 12 & 78 \\
$\mathbf{4 e}$ & $2,4-\mathrm{Cl}_{2} \mathrm{C}_{6} \mathrm{H}_{3}$ & $4-\mathrm{ClC}_{6} \mathrm{H}_{4}$ & 10 & 76 \\
$\mathbf{4 f}$ & $3-\mathrm{O}_{2} \mathrm{NC}_{6} \mathrm{H}_{3}$ & $4-\mathrm{ClC}_{6} \mathrm{H}_{4}$ & 12 & 62 \\
$\mathbf{4 g}$ & $2,4-\mathrm{Cl}_{2} \mathrm{C}_{6} \mathrm{H}_{3}$ & $\mathrm{Ph}$ & 8 & 73 \\
4h & $4-\mathrm{BrC}_{6} \mathrm{H}_{4}$ & $\mathrm{Ph}$ & 10 & 56 \\
\hline
\end{tabular}

a Refluxing conditions with $\mathbf{1} /(\mathrm{BoC})_{2} \mathrm{O} / \mathrm{NiCl}_{2} / \mathrm{Nal}=1: 1: 1: 1$.

b Isolated yields.

the same as that of iodide, also involving a regio- and stereocontrolled ring-expansion process. To test the scope of this novel method, substituted ketoaziridines $\mathbf{1}$ were reacted with benzoyl chloride in the presence of nickel(II) chloride and sodium iodide, which resulted in the production of oxazolines $\mathbf{7}$ in moderate to good yields (Table 3); no trace of another regioisomer was detected. All the products were characterized by ${ }^{1} \mathrm{H}$ NMR, ${ }^{13} \mathrm{C}$ NMR and IR spectroscopy.

A striking feature of this process is that $\mathrm{N}$-acyl-substituted ketoaziridines, in the presence of nickel(II) chloride and sodium iodide, result in oxazolines 7 via a regio- and
Table 3 Substrate Scope for the Ring Expansion of $\mathrm{N}$-H Ketoaziridines 1 to the Corresponding Oxazolines 7

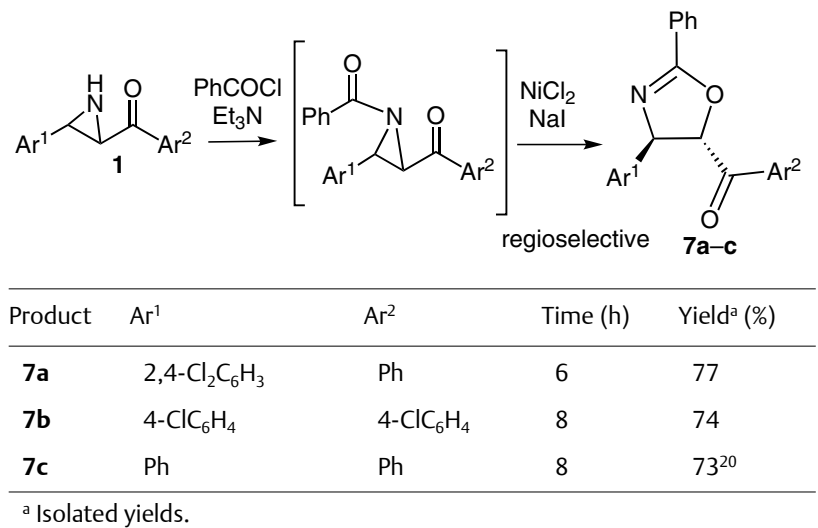

stereocontrolled ring-expansion reaction, while $N$-Boc-substituted ketoaziridines produce tert-butyl 1,3-diaryl-3-oxopropylcarbamates 4 through a reductive ring-opening reaction. This shows the influence of the $\mathrm{N}$-substituent on the ring expansion or reductive ring opening of ketoaziridines.

The exact mechanism of the novel reductive ring-opening reaction is not clear. Research in this respect is under way.

In conclusion, we have disclosed the highly efficient, reductive ring opening of $\mathrm{N}-\mathrm{H}$ ketoaziridines under refluxing conditions via a regioselective reaction promoted with $(\mathrm{Boc})_{2} \mathrm{O}$ in the presence of nickel(II) chloride and sodium iodide as an inexpensive reagent system.

All yields refer to isolated products after purification by column chromatography or by distillation under reduced pressure. Products were characterized by comparison with authentic samples (IR and ${ }^{1} \mathrm{H}$ NMR spectra, TLC, melting and boiling points). NMR spectra were recorded in $\mathrm{CDCl}_{3}$ on a Bruker AMX-400 spectrometer $\left({ }^{1} \mathrm{H}\right.$ NMR at $400 \mathrm{MHz}$ and ${ }^{13} \mathrm{C}$ NMR at $100 \mathrm{MHz}$ ) with chemical shift values $(\delta)$ in ppm downfield from TMS. IR spectra were recorded on a JASCO FT/IR-6300 spectrometer. All solvents used were dried and distilled according to standard procedures.

tert-Butyl 1,3-Diaryl-3-oxopropylcarbamates 4a-h (Table 2); General Procedure

$\mathrm{NiCl}_{2}(1.0 \mathrm{mmol})$ and $\mathrm{NaI}(1.0 \mathrm{mmol})$ were added to a solution of the ketoaziridine $1(1.0 \mathrm{mmol})$ and $(\mathrm{Boc})_{2} \mathrm{O}(1.0 \mathrm{mmol})$ in acetone $(15$ $\mathrm{mL}$ ). The mixture was refluxed for 8-12 h. The crude product was purified by column chromatography (silica gel; EtOAc-hexane, 2:5) to provide the desired corresponding tert-butyl 3-oxopropylcarbamate 4; yield: $56-78 \%$.

tert-Butyl 1,3-Bis(4-chlorophenyl)-3-oxopropylcarbamate (4a) Yield: $279 \mathrm{mg}$ (71\%); white solid; mp $140-142^{\circ} \mathrm{C}$. IR (KBr): 3280, 1685, 1596, 1452, 1321, $1228 \mathrm{~cm}^{-1}$. 
${ }^{1} \mathrm{H}$ NMR $\left(400 \mathrm{MHz}, \mathrm{CDCl}_{3}\right): \delta=1.34(\mathrm{~s}, 9 \mathrm{H}), 3.31(\mathrm{dd}, J=16.8,6.1 \mathrm{~Hz}, 1$ H), 3.55 (br d, 1 H), 5.12 (br d, 1 H), 5.48 (br, 1 H), 7.20 (m, 4 H), 7.35 $(\mathrm{d}, J=8.6 \mathrm{~Hz}, 2 \mathrm{H}), 7.76(\mathrm{~d}, J=8.6 \mathrm{~Hz}, 2 \mathrm{H})$.

${ }^{13} \mathrm{C}$ NMR $\left(100 \mathrm{MHz}, \mathrm{CDCl}_{3}\right): \delta=28.3,44.0,50.8,79.9,127.7,128.7$, $129.0,129.1,129.5,133.1,134.8,140.0,155.1,196.6$.

Anal. Calcd for $\mathrm{C}_{20} \mathrm{H}_{21} \mathrm{Cl}_{2} \mathrm{NO}_{3}$ : C, 60.92; H, 5.37; N, 3.55. Found: C, $60.89 ; \mathrm{H}, 5.39 ; \mathrm{N}, 3.59$.

\section{tert-Butyl 1-(3-Nitrophenyl)-3-oxo-3-phenylpropylcarbamate (4b)}

Yield: $251 \mathrm{mg}$ (68\%); white solid; $\mathrm{mp} 138-140{ }^{\circ} \mathrm{C}$.

IR (KBr): 3310, 1688, 1594, 1535, 1458, 1324, $1350 \mathrm{~cm}^{-1}$.

${ }^{1} \mathrm{H} \mathrm{NMR}\left(400 \mathrm{MHz}, \mathrm{CDCl}_{3}\right): \delta=1.35(\mathrm{~s}, 9 \mathrm{H}), 3.43$ (dd, $J=17.3,5.4 \mathrm{~Hz}, 1$ H), 3.63 (br d, 1 H), 5.26 (br d, 1 H), 5.78 (br, 1 H), 7.43 (m, 3 H), 7.50 $(\mathrm{td}, J=7.4,1.3 \mathrm{~Hz}, 1 \mathrm{H}), 7.64(\mathrm{~d}, J=7.1 \mathrm{~Hz}, 1 \mathrm{H}), 7.78(\mathrm{~m}, 2 \mathrm{H}), 8.11$ (dd, $J=8.2,1.3 \mathrm{~Hz}, 1 \mathrm{H}), 8.14(\mathrm{t}, J=1.9 \mathrm{~Hz}, 1 \mathrm{H})$.

${ }^{13} \mathrm{C}$ NMR $\left(100 \mathrm{MHz}, \mathrm{CDCl}_{3}\right): \delta=28.3,43.6,50.5,80.2,121.3,122.3$, 128.0, 128.8, 129.5, 129.6, 132.8, 133.8, 136.2, 148.3, 155.2, 189.8.

Anal. Calcd for $\mathrm{C}_{20} \mathrm{H}_{22} \mathrm{~N}_{2} \mathrm{O}_{5}$ : C, 64.85; H, 5.99; N, 7.56. Found: C, 64.70; H, 6.19; N, 7.69.

\section{tert-Butyl 1-(4-Chlorophenyl)-3-oxo-3-phenylpropylcarbamate} (4c)

Yield: $262 \mathrm{mg}$ (73\%); white solid; mp $142-144{ }^{\circ} \mathrm{C}$.

IR (KBr): 3310, 1688, 1594, 1459, 1331, $1221 \mathrm{~cm}^{-1}$.

${ }^{1} \mathrm{H}$ NMR ( $400 \mathrm{MHz}, \mathrm{CDCl}_{3}$ ): $\delta=1.34(\mathrm{~s}, 9 \mathrm{H}), 3.34(\mathrm{dd}, J=16.8,5.8 \mathrm{~Hz}, 1$ H), 3.56 (br d, $1 \mathrm{H}), 5.14$ (br d, $1 \mathrm{H}), 5.59$ (br, $1 \mathrm{H}), 7.22(\mathrm{~m}, 4 \mathrm{H}), 7.35$ $(\mathrm{t}, J=7.1 \mathrm{~Hz}, 2 \mathrm{H}), 7.47(\mathrm{t}, J=7.1 \mathrm{~Hz}, 1 \mathrm{H}), 7.78(\mathrm{t}, J=7.5 \mathrm{~Hz}, 2 \mathrm{H})$.

${ }^{13} \mathrm{C}$ NMR $\left(100 \mathrm{MHz}, \mathrm{CDCl}_{3}\right): \delta=28.3,43.9,50.7,79.8,127.7,128.0$, $128.7,128.8,132.9,133.5,136.5,140.3,155.1,197.8$.

Anal. Calcd for $\mathrm{C}_{20} \mathrm{H}_{22} \mathrm{ClNO}_{3}$ : C, 66.75; $\mathrm{H}, 6.16 ; \mathrm{N}, 3.89$. Found: $\mathrm{C}$, $66.64 ; \mathrm{H}, 6.24 ; \mathrm{N}, 3.94$.

\section{tert-Butyl 3-0xo-1,3-diphenylpropylcarbamate (4d)}

Yield: $253 \mathrm{mg}$ (78\%); white solid; $\mathrm{mp} 135-137^{\circ} \mathrm{C}$.

IR (KBr): 3289, 1681, 1591, 1449, 1318, $1221 \mathrm{~cm}^{-1}$.

${ }^{1} \mathrm{H}$ NMR (400 MHz, $\mathrm{CDCl}_{3}$ ): $\delta=1.34(\mathrm{~s}, 9 \mathrm{H}), 3.36$ (dd, $J=16.6,6.1 \mathrm{~Hz}, 1$ H), 3.57 (br d, $1 \mathrm{H}), 5.17$ (br d, $1 \mathrm{H}), 5.52(\mathrm{br}, 1 \mathrm{H}), 7.16(\mathrm{t}, J=6.9 \mathrm{~Hz}, 1$ H), $7.22(\mathrm{~m}, 4 \mathrm{H}), 7.34(\mathrm{t}, J=7.8 \mathrm{~Hz}, 2 \mathrm{H}), 7.46(\mathrm{t}, J=7.4 \mathrm{~Hz}, 1 \mathrm{H}), 7.80$ (t, $J=7.1 \mathrm{~Hz}, 2 \mathrm{H})$.

${ }^{13} \mathrm{C}$ NMR $\left(100 \mathrm{MHz}, \mathrm{CDCl}_{3}\right): \delta=28.3,44.2,51.4,79.6,126.3,127.3$, $128.1,128.6,128.7,133.3,136.7,141.6,155.2,190.2$.

Anal. Calcd for $\mathrm{C}_{20} \mathrm{H}_{23} \mathrm{NO}_{3}$ : C, 73.82; H, 7.12; N, 4.30. Found: C, 73.70; $\mathrm{H}, 7.15 ; \mathrm{N}, 4.41$.

\section{tert-Butyl 3-(4-Chlorophenyl)-1-(2,4-dichlorophenyl)-3-oxopro- pylcarbamate (4e)}

Yield: $324 \mathrm{mg}$ (76\%); white solid; $\mathrm{mp} 142-144{ }^{\circ} \mathrm{C}$.

IR (KBr): 3288, 1685, 1599, 1452, 1325, $1220 \mathrm{~cm}^{-1}$.

${ }^{1} \mathrm{H}$ NMR $\left(400 \mathrm{MHz}, \mathrm{CDCl}_{3}\right): \delta=1.49(\mathrm{~s}, 9 \mathrm{H}), 3.33(\mathrm{dd}, J=16.7,5.4 \mathrm{~Hz}, 1$ H), 3.55 (br d, $1 \mathrm{H}), 5.40$ (br d, $1 \mathrm{H}$ ), 5.87 (br, $1 \mathrm{H}), 7.14$ (d, J = 8.6 Hz, 1 H), $7.27(\mathrm{~m}, 3 \mathrm{H}), 7.33(\mathrm{t}, J=8.8 \mathrm{~Hz}, 2 \mathrm{H}), 7.74(\mathrm{~d}, J=8.6 \mathrm{~Hz}, 1 \mathrm{H})$.

${ }^{13} \mathrm{C}$ NMR $\left(100 \mathrm{MHz}, \mathrm{CDCl}_{3}\right): \delta=28.3,43.9,50.7,79.8,127.8,128.0$, 128.3, 128.7, 128.8, 128.9, 132.9, 133.5, 136.5, 140.3, 155.1, 197.8.

Anal. Calcd for $\mathrm{C}_{20} \mathrm{H}_{20} \mathrm{Cl}_{3} \mathrm{NO}_{3}$ : C, 56.03; $\mathrm{H}, 4.70 ; \mathrm{N}$, 3.27. Found: $\mathrm{C}$, $56.20 ; \mathrm{H}, 4.85 ; \mathrm{N}, 3.39$.
tert-Butyl 3-(4-Chlorophenyl)-1-(3-nitrophenyl)-3-oxopropylcarbamate (4f)

Yield: $250 \mathrm{mg}$ (62\%); red oil.

IR (KBr): 3294, 1681, 1591, 1355, 1452, 1345, $1228 \mathrm{~cm}^{-1}$.

${ }^{1} \mathrm{H} \mathrm{NMR}$ (400 MHz, $\mathrm{CDCl}_{3}$ ): $\delta=1.35$ (s, $9 \mathrm{H}$ ), 3.41 (dd, $J=17.1,5.7 \mathrm{~Hz}, 1$ H), 3.61 (br d, $1 \mathrm{H}), 5.27$ (br d, $1 \mathrm{H}), 5.67(\mathrm{br}, 1 \mathrm{H}), 7.35(\mathrm{~d}, J=8.7 \mathrm{~Hz}, 2$ H), 7.41-7.45 (d, $J=8.0 \mathrm{~Hz}, 1 \mathrm{H}), 7.63(\mathrm{~d}, J=7.2 \mathrm{~Hz}, 1 \mathrm{H}), 7.78(\mathrm{~d}, J=8.7$ $\mathrm{Hz}, 2 \mathrm{H}$ ), 8.02 (dd, $J=8.0,1.2 \mathrm{~Hz}, 1 \mathrm{H}$ ), 8.40 (s, $1 \mathrm{H}$ ).

${ }^{13} \mathrm{C}$ NMR $\left(100 \mathrm{MHz}, \mathrm{CDCl}_{3}\right): \delta=28.3,43.4,50.5,80.3,121.2,122.4$, 122.9, 129.1, 129.4, 129.5, 132.8, 134.5, 140.3, 148.4, 155.1, 193.8.

Anal. Calcd for $\mathrm{C}_{20} \mathrm{H}_{21} \mathrm{ClN}_{2} \mathrm{O}_{5}$ : C, 59.53; $\mathrm{H}, 5.23 ; \mathrm{N}, 6.92$. Found: $\mathrm{C}$, 59.53; H, 5.41; N, 6.84.

\section{tert-Butyl 1-(2,4-Dichlorophenyl)-3-oxo-3-phenylpropylcarba- mate (4g)}

Yield: $287 \mathrm{mg}$ (73\%); white solid; $\mathrm{mp} 131-133^{\circ} \mathrm{C}$.

IR (KBr): 3299, 1683, 1584, 1451, 1329, $1220 \mathrm{~cm}^{-1}$.

${ }^{1} \mathrm{H}$ NMR $\left(400 \mathrm{MHz}, \mathrm{CDCl}_{3}\right): \delta=1.34(\mathrm{~s}, 9 \mathrm{H}), 3.32(\mathrm{dd}, J=16.82,6.13$ Hz, $1 \mathrm{H}$ ), 3.65 (br d, $1 \mathrm{H}), 5.13$ (br d, $1 \mathrm{H}), 5.54$ (br, $1 \mathrm{H}), 7.19(\mathrm{~m}, 3 \mathrm{H})$, $7.34(\mathrm{t}, J=7.67 \mathrm{~Hz}, 2 \mathrm{H}), 7.49(\mathrm{t}, J=7.2 \mathrm{~Hz}, 1 \mathrm{H}), 7.71(\mathrm{~d}, J=7.48 \mathrm{~Hz}, 2$ $\mathrm{H})$.

${ }^{13} \mathrm{C}$ NMR $\left(100 \mathrm{MHz}, \mathrm{CDCl}_{3}\right): \delta=28.3,43.6,50.5,80.3,121.2,122.4$, $129.1,129.3,129.5,129.7,132.8,140.3,148.4,155.1,193.8$.

Anal. Calcd for $\mathrm{C}_{20} \mathrm{H}_{21} \mathrm{Cl}_{2} \mathrm{NO}_{3}$ : C, 60.92; $\mathrm{H}, 5.37 ; \mathrm{N}, 3.55$. Found: C, $60.84 ; \mathrm{H}, 5.46 ; \mathrm{N}, 3.61$.

\section{tert-Butyl 1-(4-Bromophenyl)-3-oxo-3-phenylpropylcarbamate} (4h)

Yield: $226 \mathrm{mg}$ (56\%); white solid; mp $137-139{ }^{\circ} \mathrm{C}$.

IR (KBr): 3288, 1684, 1593, 1458, 1325, $1222 \mathrm{~cm}^{-1}$.

${ }^{1} \mathrm{H} \mathrm{NMR}\left(400 \mathrm{MHz}, \mathrm{CDCl}_{3}\right): \delta=1.32(\mathrm{~s}, 9 \mathrm{H}), 3.32(\mathrm{dd}, J=16.7,5.8 \mathrm{~Hz}, 1$ H), 3.55 (br d, 1 H), 5.13 (br d, $1 \mathrm{H}), 5.59$ (br, $1 \mathrm{H}), 7.30-7.55$ (m, $4 \mathrm{H}$ ), $7.37(\mathrm{t}, J=7.2 \mathrm{~Hz}, 2 \mathrm{H}), 7.75(\mathrm{t}, J=7.2 \mathrm{~Hz}, 1 \mathrm{H}), 7.8(\mathrm{~d}, J=7.6 \mathrm{~Hz}, 2 \mathrm{H})$.

${ }^{13} \mathrm{C}$ NMR $\left(100 \mathrm{MHz}, \mathrm{CDCl}_{3}\right): \delta=28.3,43.9,50.7,79.8,127.7,128.1$, $128.7,128.8,132.9,133.5,136.5,140.3,155.1,197.8$.

Anal. Calcd for $\mathrm{C}_{20} \mathrm{H}_{22} \mathrm{BrNO}_{3}$ : C, 59.42; $\mathrm{H}, 5.48 ; \mathrm{N}, 3.46$. Found: C, 59.38; H, 5.61; N, 3.51 .

\section{X-ray Crystal Structure Analysis for 4a}

Formula: $\mathrm{C}_{20} \mathrm{H}_{21} \mathrm{Cl}_{2} \mathrm{NO}_{3}, M=394.28$, yellow crystal $0.50 \times 0.15 \times 0.01$ $\mathrm{mm}, a=11.6327(13) \AA, b=19.181(2) \AA, c=9.9585(11) \AA, \quad V=$ 2039.6(4) $\AA^{3}, \rho_{\text {calcd }}=1.284 \mathrm{mg} \cdot \mathrm{cm}^{-3}, \mu=0.337 \mathrm{~mm}^{-1}, Z=4$, monoclinic, $\lambda=0.71073 \AA, T=296 \mathrm{~K}, \omega$ and $\phi$ scans, 3580 reflections collected $( \pm h$, $\pm k, \pm l)[I \geq 2 \sigma(I)], 1959$ independent $\left(R_{\mathrm{int}}=0.1319\right)$ and 1852 observed reflections $[I \geq 2 \sigma(I)], 242$ refined parameters, $R=0.0544, w R^{2}=$ 0.1260 , max residual electron density $0.24(-0.29)$ e. $\AA^{-3}$. Flack parameter 0.0040(6). The data set was collected with a Bruker SMART APEX CCD area detector diffractometer, absorption correction: multi-scan $\left(\right.$ SADABS $^{35}$ ), at the School of Chemical Sciences and Food Technology, University Kebangsaan Malaysia.

\section{Oxazolines 7a,b (Table 3); General Procedure}

Benzoyl chloride $(1.0 \mathrm{mmol})$ was added dropwise to a solution of an aziridine $1(1.0 \mathrm{mmol})$ and $\mathrm{Et}_{3} \mathrm{~N}(2.0 \mathrm{mmol})$ in $\mathrm{CH}_{2} \mathrm{Cl}_{2}(10 \mathrm{~mL})$ at $0{ }^{\circ} \mathrm{C}$. The mixture was stirred for $1.5 \mathrm{~h}$ at this temperature and for $4 \mathrm{~h}$ at r.t., and then rinsed with $\mathrm{H}_{2} \mathrm{O}(2 \times 10 \mathrm{~mL})$. The organic layer was dried over anhydrous $\mathrm{Na}_{2} \mathrm{SO}_{4}$ and concentrated under reduced pressure. 
Acetone $(15 \mathrm{~mL}), \mathrm{NaI}(1 \mathrm{mmol})$ and $\mathrm{NiCl}_{2}(1 \mathrm{mmol})$ were added to the crude product and the mixture was stirred at $50{ }^{\circ} \mathrm{C}$ for $6 \mathrm{~h}$. Then, the mixture was rinsed with $\mathrm{H}_{2} \mathrm{O}(2 \times 10 \mathrm{~mL})$, and the organic layer was separated and dried with anhydrous $\mathrm{Na}_{2} \mathrm{SO}_{4}$. Evaporation of the solvent under reduced pressure and subsequent purification of the residue by column chromatography (silica gel; EtOAc-hexane, 1:4) provided the corresponding oxazoline 7 .

\section{trans-5-Benzoyl-4-(2,4-dichlorophenyl)-2-phenyl-2-oxazoline}

(7a)

Yield: $304 \mathrm{mg}$ (77\%); white solid; $\mathrm{mp} 128-130{ }^{\circ} \mathrm{C}$.

IR (KBr): 3066, 2907, 1680, 1652, 1591, 1481, 1261, 760, 707, $695 \mathrm{~cm}^{-1}$. ${ }^{1} \mathrm{H} \mathrm{NMR}\left(400 \mathrm{MHz}, \mathrm{CDCl}_{3}\right): \delta=5.58(\mathrm{~d}, J=6.7 \mathrm{~Hz}, 1 \mathrm{H}), 5.75(\mathrm{~d}, J=6.7$ $\mathrm{Hz}, 1 \mathrm{H}), 7.36-7.67$ (m, $9 \mathrm{H}), 7.99$ (d, $J=7.3 \mathrm{~Hz}, 2 \mathrm{H}), 8.14$ (d, $J=7.3 \mathrm{~Hz}$, $2 \mathrm{H})$.

${ }^{13} \mathrm{C}$ NMR $\left(100 \mathrm{MHz}, \mathrm{CDCl}_{3}\right): \delta=74.3,87.4,127.4,127.5,128.3,128.6$, $128.8,129.2,129.3,129.4,129.5,132.3,134.4,134.6,141.1,164.3$, 194.9.

Anal. Calcd for $\mathrm{C}_{22} \mathrm{H}_{15} \mathrm{Cl}_{2} \mathrm{NO}_{2}$ : C, 66.68; $\mathrm{H}, 3.82 ; \mathrm{N}, 3.53$. Found: $\mathrm{C}$, $66.57 ; \mathrm{H}, 3.84 ; \mathrm{N}, 3.47$.

\section{trans-5-(4-Chlorobenzoyl)-4-(4-chlorophenyl)-2-phenyl-2-oxazo-} line (7b)

Yield: $292 \mathrm{mg}$ (74\%); white solid; $\mathrm{mp} 135-137^{\circ} \mathrm{C}$.

IR (KBr): 3058, 2921, 1684, 1650, 1594, 1483, 1266, 761, 709, $696 \mathrm{~cm}^{-1}$. ${ }^{1} \mathrm{H} \mathrm{NMR}\left(400 \mathrm{MHz}, \mathrm{CDCl}_{3}\right): \delta=5.50(\mathrm{~d}, J=6.5 \mathrm{~Hz}, 1 \mathrm{H}), 5.70(\mathrm{~d}, J=6.5$ $\mathrm{Hz}, 1 \mathrm{H}), 7.30-7.70(\mathrm{~m}, 9 \mathrm{H}), 7.90(\mathrm{~d}, J=7.3 \mathrm{~Hz}, 2 \mathrm{H}), 8.10(\mathrm{~d}, J=7.2 \mathrm{~Hz}$, $2 \mathrm{H})$.

${ }^{13} \mathrm{C}$ NMR $\left(100 \mathrm{MHz}, \mathrm{CDCl}_{3}\right): \delta=74.3,87.4,125.6,127.5,128.8,129.2$, 129.3, 129.4, 129.5, 130.7, 134.4, 134.6, 138.7, 141.1, 163.3, 194.6.

Anal. Calcd for $\mathrm{C}_{22} \mathrm{H}_{15} \mathrm{Cl}_{2} \mathrm{NO}_{2}$ : C, 66.68; $\mathrm{H}, 3.82 ; \mathrm{N}, 3.53$. Found: $\mathrm{C}$, $66.61 ; \mathrm{H}, 3.79 ; \mathrm{N}, 3.74$.

\section{(E)-1,3-Bis(4-chlorophenyl)prop-2-en-1-one (3a)}

Yellow crystals; mp $156-158^{\circ} \mathrm{C}$.

IR (KBr): 3041, 1655, 1597, 883, 774, 699, $682 \mathrm{~cm}^{-1}$.

${ }^{1} \mathrm{H} \mathrm{NMR}\left(400 \mathrm{MHz}, \mathrm{CDCl}_{3}\right): \delta=7.50(\mathrm{~m}, 2 \mathrm{H}), 7.53(\mathrm{dd}, J=7.6,1.8 \mathrm{~Hz}, 1$ H), $7.60(\mathrm{~m}, 2 \mathrm{H}), 7.73(\mathrm{~m}, 2 \mathrm{H}), 7.76(\mathrm{~d}, J=15.5 \mathrm{~Hz}, 1 \mathrm{H}), 8.20$ (dd, $J=$ 7.5, $2.2 \mathrm{~Hz}, 2 \mathrm{H})$.

${ }^{13} \mathrm{C}$ NMR $\left(100 \mathrm{MHz}, \mathrm{CDCl}_{3}\right): \delta=122.2,128.4,128.5,129.3,129.4$, $132.3,133.9,136.8,139.1,144.1,189.8$.

Anal. Calcd for $\mathrm{C}_{15} \mathrm{H}_{10} \mathrm{Cl}_{2} \mathrm{O}$ : C, 65.01; H, 3.64. Found: C, 65.08; $\mathrm{H}, 3.69$.

\section{Acknowledgment}

We are thankful to the Research Council of Shahrekord University for supporting this work, and to the Universiti Kebangsaan Malaysia Instrumentation Center (UKM-DIP 2012-11) for the X-ray analysis.

\section{Supporting Information}

Supporting information for this article is available online at http://dx.doi.org/10.1055/s-0034-1379029.

\section{References}

(1) Hu, X. E. Tetrahedron 2004, 60, 2701.

(2) Casimir, J. R.; Turetta, C.; Ettouati, L.; Paris, J. Tetrahedron Lett. 1995, 36, 4797.

(3) David, F. A.; Reddy, G. V.; Liang, C.-H. Tetrahedron Lett. 1997, 38, 5139.

(4) Fiedler, E.; Fiedler, H.-P.; Gerhard, A.; Keller-Schierlein, W.; König, W. A.; Zähner, H. Arch. Microbiol. 1976, 107, 249.

(5) Tiwari, A. K.; Kumbhare, R. M.; Agawane, S. B.; Ali, A. Z.; Kumar, K. V. Bioorg. Med. Chem. Lett. 2008, 18, 4130.

(6) For general reviews, see: Enantioselective Synthesis of $\beta$-Amino Acids; Juaristi, E., Ed.; Wiley-VCH: Weinheim, 1997.

(7) Cardillo, G.; Tolomelli, A.; Tomasini, C. J. Org. Chem. 1996, 61, 8651.

(8) Davis, F. A.; Zhang, Y.; Rao, A.; Zhang, Z. Tetrahedron 2001, 57, 6345.

(9) Hwang, G.-I.; Chung, J.-H.; Lee, W. K. J. Org. Chem. 1996, 61, 6183.

(10) Higashiyama, K.; Matsumura, M.; Shiogama, A.; Yamauchi, T.; Ohmiya, S. Heterocycles 2002, 58, 85.

(11) Dauban, P.; Dodd, R. H. Tetrahedron Lett. 1998, 39, 5739.

(12) Kim, S.; Jung, M. S.; Cho, C. H.; Schiesser, C. H. Tetrahedron Lett. 2001, 42, 943.

(13) Chandrasekhar, S.; Ahmed, M. Tetrahedron Lett. 1999, 40, 9325.

(14) Davis, A. L.; Korous, A. A.; Hartel, A. M. Tetrahedron Lett. 2013, 54, 3673.

(15) Pak, C. S.; Kim, T. H.; Ha, S. J. J. Org. Chem. 1998, 63, 10006.

(16) Almena, J.; Foubelo, F.; Yus, M. J. Org. Chem. 1994, 59, 3210.

(17) Shimizu, M.; Kurokawa, H.; Nishiura, S.; Hachiya, I. Heterocycles 2006, 70, 57.

(18) Reynolds, S. C.; Wengryniuk, S. E.; Hartel, A. M. Tetrahedron Lett. 2007, 48, 6751.

(19) Larraufie, M.-H.; Pellet, R.; Fensterbank, L.; Goddard, J.-P.; Lacote, E.; Malacria, M.; Ollivier, C. Angew. Chem. Int. Ed. 2011, $50,4463$.

(20) Samimi, H. A.; Mamaghani, M.; Tabatabaeian, K. J. Heterocycl. Chem. 2008, 45, 1765.

(21) Mamaghani, M.; Tabatabaeian, K.; Samimi, H. A. Z. Kristallogr. New Cryst. Struct. 2008, 223, 390.

(22) Samimi, H. A.; Shams, Z. J. Iran. Chem. Soc. 2014, 11, 979.

(23) Samimi, H. A.; Mohammadi, S. Synlett 2013, 24, 223.

(24) Samimi, H. A.; Mohammadi, S. J. Iran. Chem. Soc. 2014, 11, 69.

(25) Samimi, H. A.; Kiyani, H.; Shams, Z. J. Chem. Res. 2013, 37, 282.

(26) Samimi, H. A.; Yamin, B. M.; Hiedari, Z.; Narimani, L. J. Heterocycl. Chem. accepted for publication.

(27) Samimi, H. A.; Yamin, B. M. J. Chem. Res. 2014, 38, 358.

(28) Tomasini, C.; Vecchione, A. Org. Lett. 1999, 1, 2153.

(29) Kump, J. E. G. In Comprehensive Organic Synthesis; Trost, B. M.; Fleming, I., Eds.; Pergamon Press: Oxford, 1991, 469.

(30) Sabitha, G.; Babu, R. S.; Rajkumar, M.; Reddy, C. S.; Yadav, J. S. Tetrahedron Lett. 2001, 42, 3955.

(31) Saha, B.; Nandy, J. P.; Shukla, S.; Siddiqui, I.; Iqbal, J.J. Org. Chem. 2002, 67, 7858.

(32) Lucarini, S.; Tomasini, C. J. Org. Chem. 2001, 66, 727.

(33) Cardillo, G.; Gentilucci, L.; Gianotti, M.; Tolomelli, A. Synlett 2000, 1309.

(34) Quinze, K.; Laurent, A.; Mison, P. J. Fluorine Chem. 1989, 44, 233.

(35) Sheldrick, G. M. SADABS A Program for Structure Determination; University of Göttingen: Germany, 1996. 